\title{
Domestic Android Application for Home Services
}

\author{
Sheetal Bandekar \\ Department of Master of \\ Computer Applications \\ KLS' Gogte Institute of Technology \\ Udyambag, Belagavi - Karnataka
}

\author{
Avril D'Silva \\ Department of Master of \\ Computer Applications \\ KLS' Gogte Institute of Technology \\ Udyambag, Belagavi - Karnataka
}

\begin{abstract}
The profuse use of Mobile Applications has made them a part and parcel of our lives, be it for personal use, corporate based task or entertainment. "Domestic Android Application for Home Services", is a Corporate based mobile application for android users that brings together the client and service providers and connects them using the GPS (Global Positioning System). Clients request for the home services and based on the location by fetching the latitude and longitude of the client, the nearest service provider is allotted to serve the client's needs. By enhancing the existing system "FacilityKart" application which does not implement GPS into its application, this application has a wide scope for integrating maps to allow drag and drop to another location, and make this application available for other mobile operating systems other than android.
\end{abstract}

\section{Keywords}

Mobile Application, Android Application, GPS (Global Positioning System).

\section{INTRODUCTION}

Convergence of information technology and communication technology has created an environment where we can access information, get services online from anywhere anytime even at the state of mobility. Such access to information and online services is possible just by the use of our finger tips through mobile applications. These mobile applications can be grouped as personal, perishable, transaction oriented, location specific, corporate and entertainment [3].

"Domestic Android Application for Home Services" is a mobile application built for Android users, which is catered to the requirements of a Client who wants to provide domestic home services online by bringing together the users and service providers. The registered users can demand a service available through the application and based on the users' location, the nearest service provider is allotted to cater the users' request for service. Locating the user and the service provider is done by using the GPS (Global Positioning System) that gives the exact location on the earth. The applications' simple and straight forward interface; instant service; feature for rating and sending feedback, makes it incredibly useful and relatively easy to use for all users.

\section{TOOLS AND TECHNOLOGIES USED \\ 2.1 Android SDK}

Android Software Development Kit is a programming package to develop mobile applications that can run on android platform. This entire package comes bundled with sample projects along with the source code, development tools, emulators and required libraries to create a full-fledged android based mobile application. These applications are written using Java as a programming language and runs on
Dalvik, a custom made virtual machine for embedded use and which runs on top of Linux Kernel [4].

\subsection{Eclipse}

Eclipse is an Integrated Development Environment (IDE) which caters the developers with a platform to develop noteworthy applications. Eclipse consists of projects on different aspects of software development. Eclipse has existed with the many declarations of the Sun Micro System, and it takes responsibility for developing Java language. Eclipse projects can run on a wide range of machines like Intel, Mac and so on. Eclipse provides for enterprise development to build frameworks of applications, device development, and rich platform for clients [5].

\subsection{Java}

Java language is used in diversified application based projects and in 1995 was released as the main core component of Sun Microsystems platform of Java. Java Virtual Machine commonly does the task of organizing applications to byte code that can help with running on a java virtual machine paying little focus to pc structural engineering. Java is secured, high level robust programming language, and it has its own run time environment .The hardware and software in which program runs is called as the platform. There are so many areas where java is currently in use like in mobile devices, games, embedded systems, system robotics and enterprising applications such as banking.

\subsection{MySQL}

MySQL is one of the most widely used RDBMS in the IT industry. SQL stands for Structured Query Language. MySQL development has made the source code available under the terms of MySQL owned by Oracle. The paid editions are also available which offer additional functionality. MySQL is used in many websites such as facebook, google, twitter, and youtube. The MySQL database is used in web applications. Open source projects use MySQL. The development code is now available to the users under the use of general public license [6].

\section{LITERATURE SURVEY}

\subsection{Existing System}

There is an existing system by name "FacilityKart" a mobile application, which is similar to the application "Domestic Android Application for Home Services". This application provides the following services: Home Cleaning, Laundry Services, Driver on Demand Services, Carpet Cleaning Services, Sofa Cleaning Services, Pest Control Services, Electrical Services, Plumbing Services, AC Services, Carpentry Services, Car Spa, PC \& Laptop Repair, Refrigerator/Microwave/Washing Machine Services, and Home Painting [7]. 


\subsection{Working of Existing System}

The user needs to select the service type from the various services that are provided in the application. The user must specify the date and time for the service to be executed and door step service is provided as per the users' information provided in the application.

\section{MODULES IN EXISTING SYSTEM 4.1 Administrator Module}

This module provides administrator related functionality. Administrator manages all information and has access rights to add delete, edit and view the data related to the bookings made etc.

\subsection{Services Module}

This module provides the details of various services that are available. A user can select the appropriate service they wish to have, depending on their convenience and location.

\subsection{User Module}

This module provides information related to various services that are being offered. For each service, the application allows the user to select the date and time for the service.

\subsection{Feedback Module}

User of this application can post their opinions, complaints and suggestions regarding the application and services to the administrator. Accordingly, the administrator can take various steps to act on the complaints and suggestions.

\section{FLAWS IN THE EXISTING SYSTEM}

Once the user enters all the details, the administrator has to manually check the user details and allot the service to the service men based on user requirements. This application does not utilize the GPS (Global Positioning System) service which is available on all android devices for identifying the location of user, who could be on mobility frequently and may require home services at different locations. If a registered user changes his location and needs service, he has to register and specify his new location.

\section{PROPOSED SOLUTION}

The drawbacks in the existing system have been overcome by implementing GPS (Global Positioning System) service. User and Service Providers need to register on this application. Once the user specifies his request for service, the users' location is fetched using GPS (Global Positioning System) that fetches the latitude and longitude. Based on his current location, the application will try to find out the nearest service provider by fetching the latitude and longitude of the service provider, and then the nearest service provider is allotted to the requested user by sending SMS (Short Message Service), to cater the users' request.

\subsection{Algorithm to fetch latitude and longitude \\ Step 1:}

if(idGpsEnabled)

\{

LocationManager.GPS_PROVIDER

\}

else

\{

No Location Data Available

\}

Step 2:

LocationListener listener $=$ new UserLocationListerner();

if(current_location!=null)

\{

latitude=current_location.getLatitude();

longitude=current_location.getLaongitude();

senddata(logged_user, (latitude), (longitude));

\}

else

\{

No Stored Location Available \}

\subsection{Algorithm to compare latitude and longitude of User and Service Provider}

Following is the pseudo code that shows how the user and service providers' latitude and longitude are compared and the service provider is allotted to the user by using the distancefrom () function available in Android Programming [2].

Step 1: Fetch the latitude and longitude of the user

Step 2: Store it on the server database

Step 3: Compare the latitude and longitude of the user and the service provider by using the distancefrom () method.

Step 4: Allot a service provider based on the comparison made.

\section{IMPLEMENTATION}

Thus by studying the flaws in the existing system and proposing a new solution, a real time Android Application has been built using the technologies mentioned and named it as "Domestic Android Application for Home Services". Following are the important screen shots of the developed application on Android Platform. 


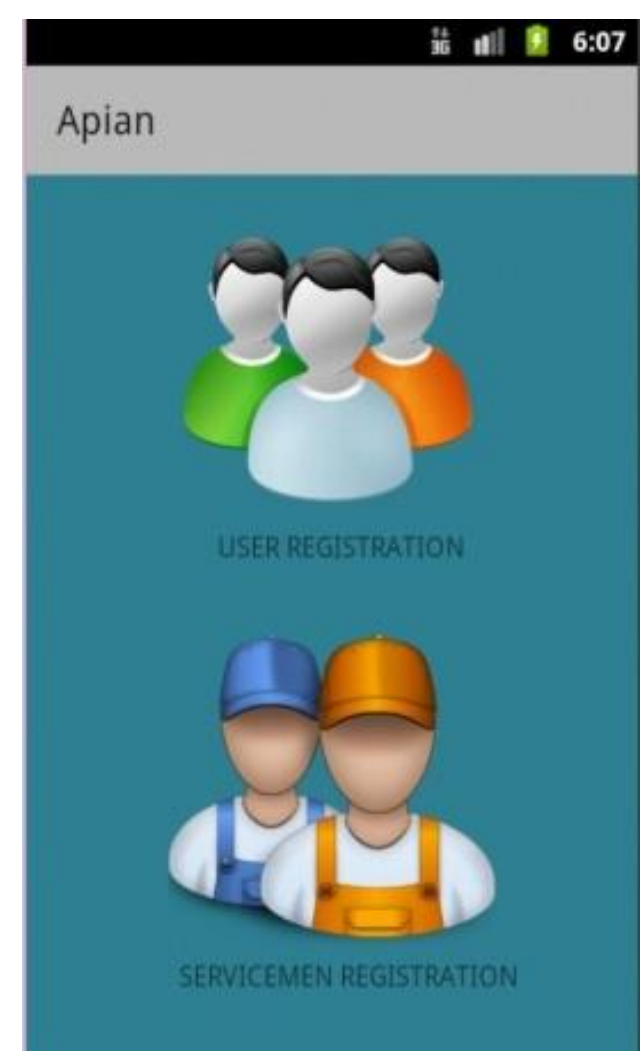

Fig 1: Category page

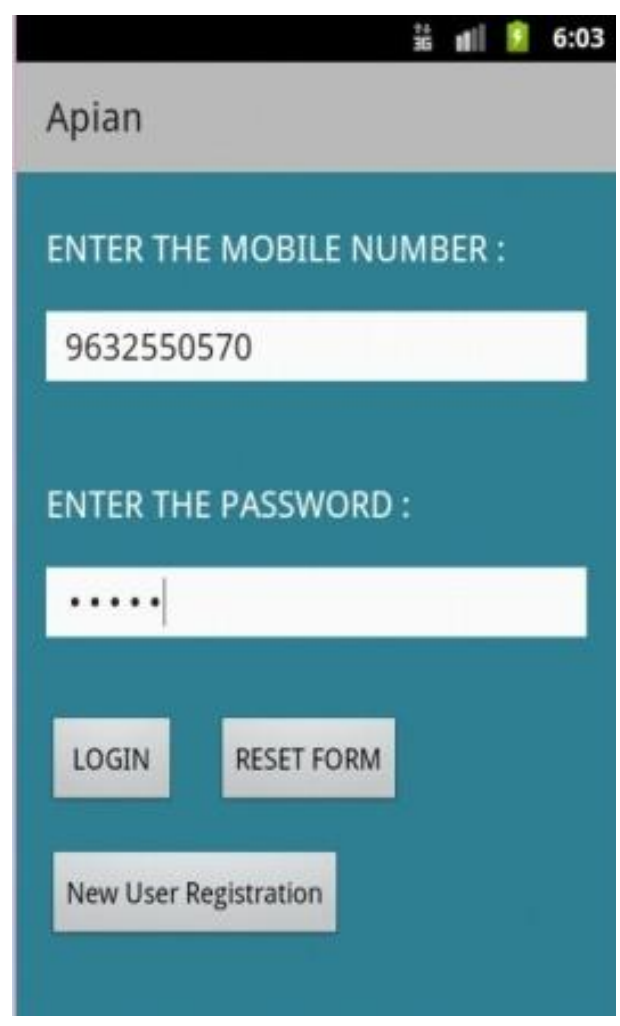

Fig 2: Login Page

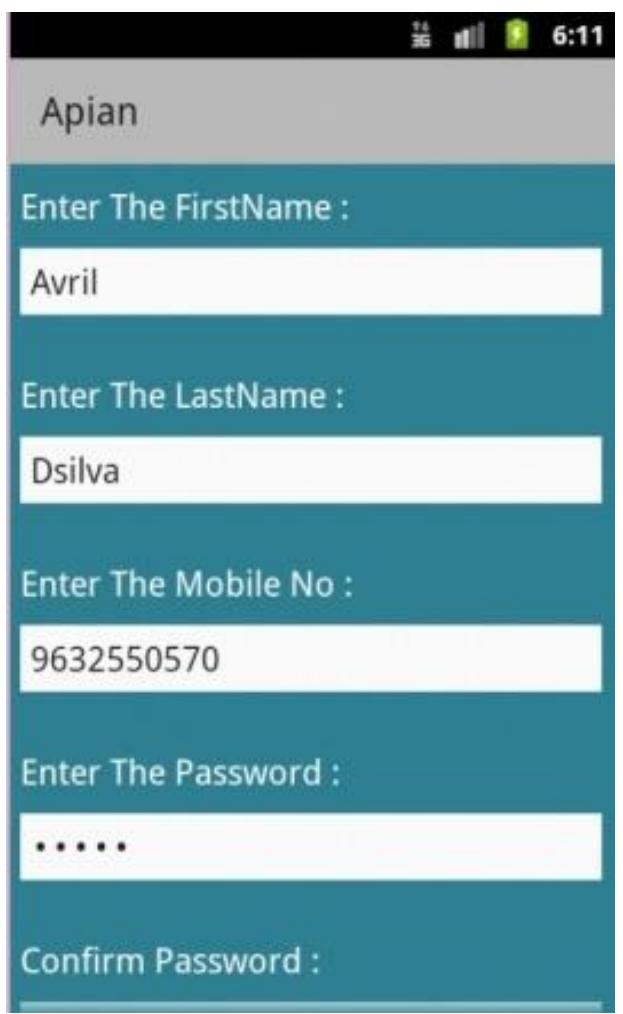

Fig 3: User Registration Page

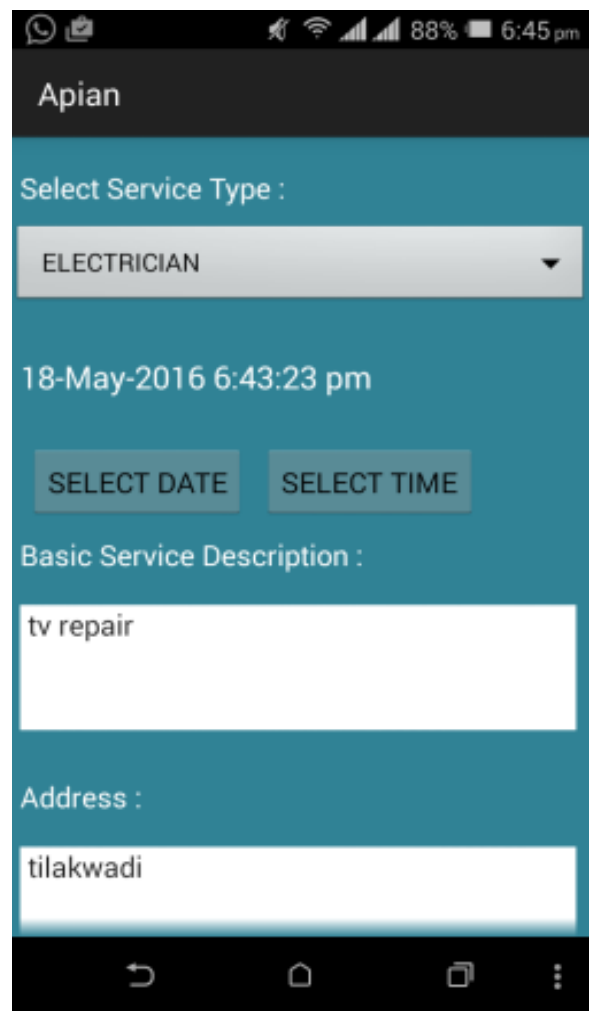

Fig 4: Add New Request Page 


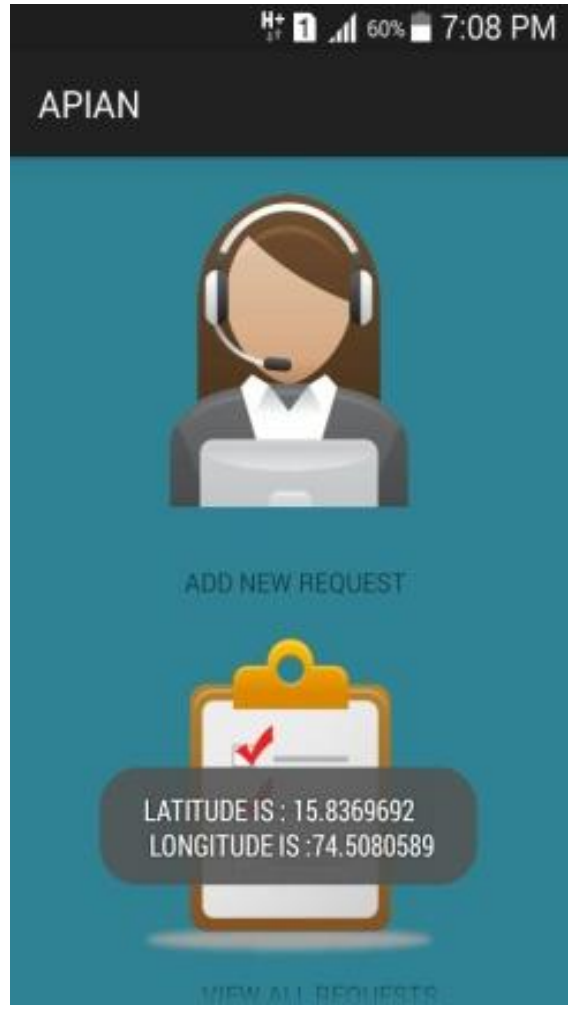

Fig 5: Fetched Latitude and Longitude of User

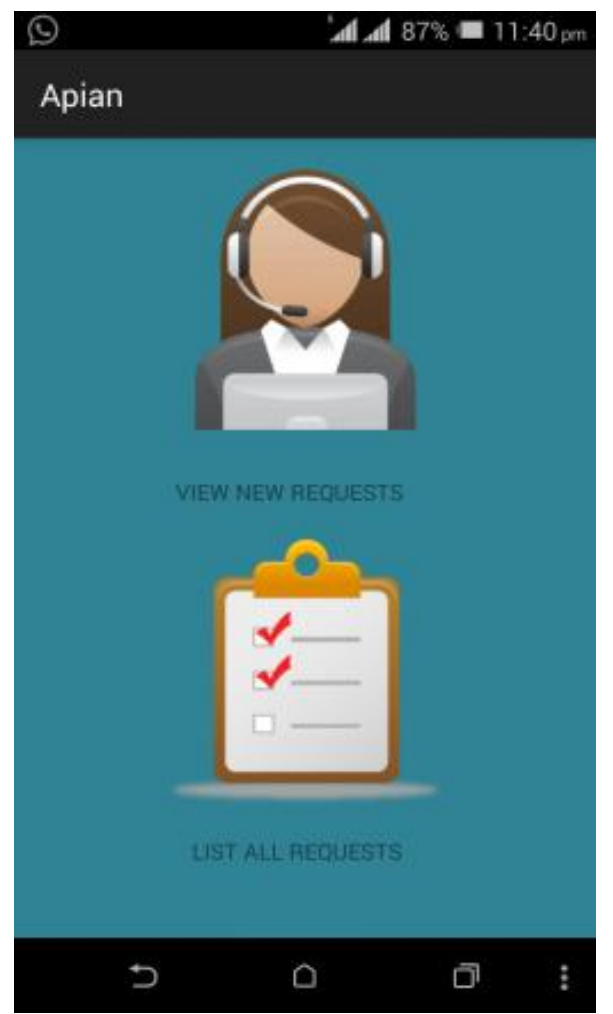

Fig 6: Service Provider Home Page

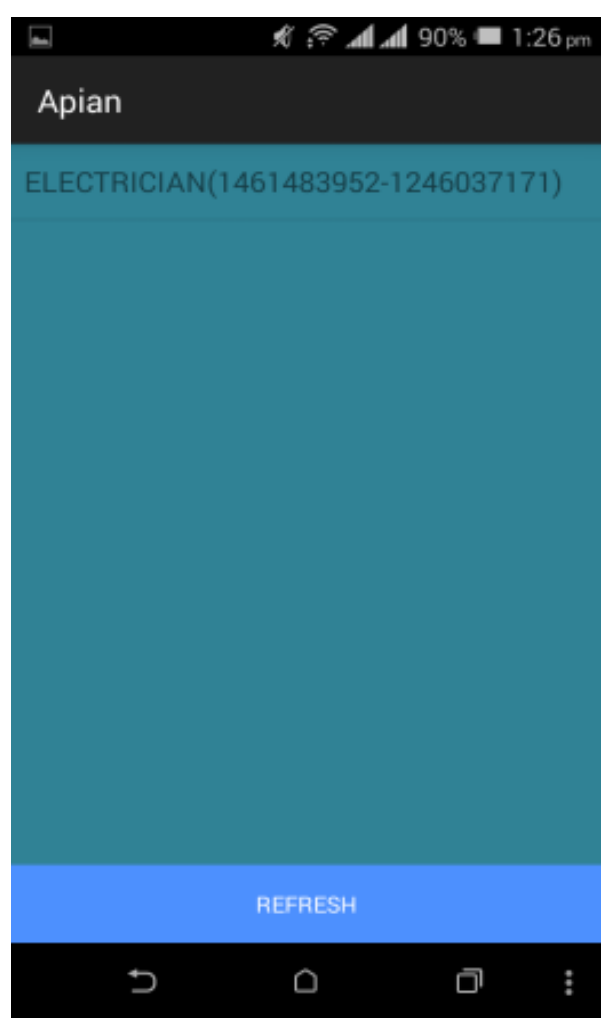

Fig 7: View Request Form

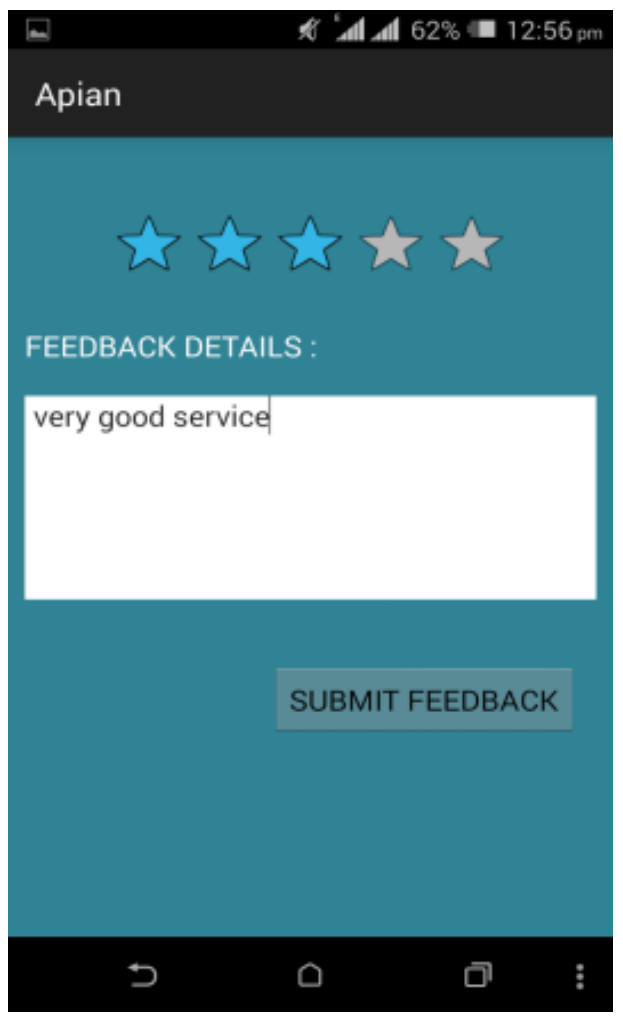

Fig 8: Feedback Form

\section{CONCLUSION}

"Domestic Android Application for Home Services" is a customized Mobile Application which uses the state of the art technologies like Android SDK (Software Development Kit), Eclipse, Java and MySQL used for Android Application Development. This application provides domestic home services where users can be served for electrical services, 
plumbing services and carpentry services. Unlike the existing "FacilityKart" application, this application uses GPS to fetch the users' location and assigns nearest service provider from his existing location dynamically. Thus this application seems to be more dynamic, effective and efficient than the existing system.

\section{FUTURE ENHANCEMENT}

This application can be further enhanced by allowing the users of the application to drag and drop to another location on the maps. This facility can be used by the user in case if the user wants the service request to be accomplished at another location than his current location. For this there is a need to integrate the maps into this application. Another useful enhancement can be made by providing more service types to the user. Lastly, since this application is built only for the Android users, this application can also be implemented for other platform like iOS and Windows.

\section{REFERENCES}

[1] Pradeep Kothari, Android Application Development, DreamTech Press.
[2] Wallace Mc Clure, Jonathan Dick and Chris Hardy, Professional Android Programming with Mono for Android, Wrox Publication.

[3] Dr. Ashok Talukder, Ms. Roopa Yavagal, Mr. Hasan Ahmed: Mobile Computing, Technology, Applications and Service Creation, 2nd Edition, Tata McGrawHill, 2010.

[4] Android SDK: A software development kit that enables to create applications for Android platform. Available: http://www.webopedia.com/TERM/A/Android_SDK.ht $\mathrm{ml}$

[5] Eclipse: Is famous for Java Integrated Development environment. Available: https://eclipse.org/ide/

[6] MySQL: Is an open source relational database management system (RDBMS). Available: https://en.wikipedia.org/wiki/MySQL.

[7] Facilitykart: Is India's' most trusted hyper local aggregator for handyman and Home need services like AC, RO, Home Appliances, Electrical, Plumbing, Carpentry etc. Available: http://facilitykart.com. 